\title{
Sentinel Lymph Node Detection by Blue Dye Versus Indocyanine Green Fluorescence Imaging in Colon Cancer
}

\author{
GABRIEL LIBERALE ${ }^{1}$, SOPHIE VANKERCKHOVE ${ }^{2}$, MARIA GOMEZ GALDON ${ }^{3}$, \\ DENIS LARSIMONT ${ }^{3}$, BISSAN AHMED ${ }^{2}$, FIKRI BOUAZZA ${ }^{1}$, MICHEL MOREAU $^{4}$, \\ ISSAM EL NAKADI ${ }^{1}$, VINCENT DONCKIER ${ }^{1}$ and PIERRE BOURGEOIS ${ }^{2}$; \\ R\&D Group for the Clinical Application of Fluorescence Imaging at the Jules Bordet Institute \\ Departments of ${ }^{1}$ Surgical Oncology, ${ }^{2}$ Nuclear Medicine and Clinic-Unit of Lymphology, ${ }^{3}$ Pathology and \\ ${ }^{4}$ Data Centre and Department of Statistics, Jules Bordet Institute, Free University of Brussels, Brussels, Belgium
}

\begin{abstract}
Background/Aim: Nodal staging is used in colorectal cancer (CRC) to determine which patients should receive adjuvant chemotherapy. The aim of this study was to evaluate the role of indocyanine green fluorescence imaging (ICG-FI) in sentinel lymph node (SLN) detection compared to the standard technique. Materials and Methods: Twenty patients with CRC admitted for elective colectomy were included (NCT01995591). Ex vivo SLN detection was performed using patent blue $(P B)$ and free ICG injected around the tumor. Results: Identification rates were $95 \%$ (19/20) for both techniques. Sensitivity was $43 \%$ for PB and $57 \%$ for ICG. Correlation between the techniques was $83 \%$. FI was more sensitive in patients with body mass index (BMI) $>25 \mathrm{~kg} / \mathrm{m}^{2}$. Serial section analysis did not allow for upstaging of patients. Conclusion: The use of ICG-FI is superior to the blue dye technique in patients with a BMI $>25$ $\mathrm{kg} / \mathrm{m}^{2}$. However, the sensitivity of ICG-FI in SLN detection remains low, with a high rate of false-negative results.
\end{abstract}

In patients with colorectal cancer (CRC), the accurate establishment of the pathological status of tumor-draining mesenteric lymph nodes (LN) is of central importance for therapeutic management. In patients with stage III disease with demonstrated LN metastases, adjuvant systemic chemotherapy is indicated, reducing the risk of recurrence and improving long-term overall survival (1). The accuracy of mesenteric

This article is freely accessible online.

Correspondence to: Dr Gabriel Liberale, Department of Surgery, Institut Jules Bordet, Université Libre de Bruxelles, 121 Blvd. de Waterloo, 1000, Brussels, Belgium. Tel: +32 25413670, Fax: +32 25413132, e-mail: Gabriel.liberale@bordet.be

Key Words: Colorectal neoplasms, fluorescence imaging, lymph node, indocyanine green, TNM staging. nodal staging depends primarily on the number of resected LN, and the seventh American Joint Committee on Cancer (AJCC) edition recommends harvesting and analysis of at least 12 to $14 \mathrm{LN}$ for correct staging (2). Despite this, 20-30\% of patients with no LN metastases demonstrated at pathology ( $\mathrm{pN} 0)$, and therefore classified as stage II, still develop recurrent disease (3). These recurrences could potentially be associated with unrecognized locoregional nodal metastases, which could lead to understaging and undertreatment.

The sentinel lymph node (SLN) is defined as the first LN draining the primary tumor. In melanoma and breast cancer, it has been shown that pathological status of the SLN is indicative of the risk of nodal metastases, thus providing an indication for lymphadenectomy when SLN pathological examination is positive and for avoiding mutilating surgery when it is negative $(4,5)$. The concept, and potential utility, of the SLN is different in CRC since mesenteric lymphadenectomy is systematically performed during surgery for the primary tumor. In patients with CRC, the particular contribution of SLN examination could be to focus the pathological analysis specifically on draining LNs in order to improve the sensitivity for cancer cell detection by the use of advanced methods, such as serial sectioning, immunohistochemistry (IHC), and molecular analyses such as reverse transcriptase polymerase chain reaction (RT-PCR) to increase pathological staging (6).

Accordingly, a recent meta-analysis concluded that the use of SLN examination allowed for nodal up-staging of patients with CRC, providing a potential benefit (7). However, the technique of SLN analysis is still not widely used in patients with CRC. This could be related to the variability of the sensitivity of techniques for SLN detection which ranges from $33 \%$ to $100 \%$, to the risk of missed metastases, and also to uncertainty related to the clinical significance and prognostic value of nodal micrometastases in CRC (7-9).

Recently, fluorescence imaging (FI) using indocyanine green (ICG) has emerged as a new technique for SLN detection in various tumors (10-12). Only few studies have 
evaluated FI in CRC (13-16). These studies have shown a potential advantage of FI compared to classical blue dye and lymphoscintigraphy techniques for the detection of deep LN, particularly in obese patients (16).

In the present work, we report our initial experience with the use of ex vivo ICG-FI in comparison to standard blue dye techniques for the detection of SLN in CRC.

\section{Materials and Methods}

Study design and patients. Patients with primary CRC admitted for elective surgery were included in the study. This was a prospective interventional study designed as an open-label, single group assigned diagnostic. The study was approved by the Investigational Review Board of the Jules Bordet Institute (CE2068) and registered at the Clinical Trials.gov Protocol Registration System (NCT01995591; https://register.clinicaltrials.gov). All patients gave their written informed consent before inclusion in the study.

The primary objective was to evaluate the feasibility of the ex vivo ICG-FI technique in patients undergoing surgery for CRC. The secondary objectives were to determine whether the ICG-FI technique increases the detection rate of SLN and to determine the sensitivity of the SLN technique for detection of LN metastases compared to the standard blue dye technique. In the study design, we assumed that the inclusion of 20 patients would be required to explore the technique's feasibility. This number corresponds to half the number of patients undergoing elective colectomy yearly for $\mathrm{CRC}$ at our Institution.

All patients underwent routine work-up to exclude extra-abdominal metastases, including thoraco-abdomino-pelvic computed tomography (CT). Fluorodeoxyglucose positron-emission tomography CT was only used in cases of suspected metastatic disease. Additional workup included complete laboratory tests, including tumor markers. Exclusion criteria were age $<18$ years, inability to give informed consent, and definite LN metastases diagnosed either pre-operatively, or intra-operatively.

Surgery. All patient surgeries were performed following standard recommendations. Right colectomy was performed by minilaparotomy unless the patient was obese, and transverse-, left- and sigmoid-colectomies were performed by laparoscopy.

Ex vivo sentinel lymph node techniques. Blue dye: Immediately after removal of the colon, the mesentery was exposed in the fresh specimen. Using different 29-Gauge tuberculin-insulin syringes, patent blue V (Guebert, Aulnay-sous-bois, France) was injected subserosally at the four cardinal points around the tumor $(0.5 \mathrm{ml} / \mathrm{site}$ of injection). The injection sites were then gently massaged for 3 to $5 \mathrm{~min}$ to stimulate the dye to flow along the lymphatic vessels. Blue LNs were identified and marked with a blue stitch. During the procedure, we avoided dissecting the mesentery in order to avoid lymphatic disruption, potentially compromising further exploration with FI.

Fluorescence technique: ICG (Pulsion, Paris, France) solution was diluted with distilled water to obtain a $0.5 \mathrm{mg} / \mathrm{ml}$ solution. ICG was injected in the same way as the blue dye. According to the molecular weight of each molecule, $774.96 \mathrm{~g} / \mathrm{mol}$ for ICG and $579.72 \mathrm{~g} / \mathrm{mol}$ for patent blue, and to avoid lymphatic obstruction, the FI technique was performed immediately after the blue dye injection and the identification of blue SLNs.
ICG-FI was performed using a dedicated near-infrared camera system (Photodynamic Eye, PDE; Hamamatsu Photonics, Hamamatsu, Japan). After FI of the mesentery, fluorescent lymphatic drainage and spots suspected to correspond to LNs were identified and marked with a green stitch (Figure 1).

Histopathology. In the pathology laboratory, LNs identified by $e x$ vivo blue dye and ICG techniques and marked with blue and green stiches were dissected for separate analyses.

Thereafter, the specimen was processed as usual using routine procedures for further pathological examination of the mesenteric LNs. Once dissected, all removed LNs were re-evaluated for blue staining by visual examination and for emitted fluorescence with the PDE camera system. Those LNs were noted as 'blue' and 'fluorescent' afterwards at the Pathology Department but were not considered to be SLNs.

SLNs were fixed and embedded with paraffin and then stained with hematoxylin and eosin (HE). SLNs that were negative with standard HE analysis (bivalve section) were further analyzed with serial sectioning. Serial sectioning was performed using three $5-\mu \mathrm{m}$ slices cut at a $150 \mu \mathrm{m}$ interval and stained with HE. If still negative, they were further analyzed with IHC using anti-pan-cytokeratin AE1/AE3/PCK26 (DAKO, Agilent techniologies, Glostrup, Denmark). All specimens were analyzed and staged according to the seventh AJCC classification (pTNM). Metastases visible after HE staining measuring 0.2 to $2 \mathrm{~mm}$ along their largest diameter were defined as 'authentic' micrometastases and thus classified as pN1. 'Occult' micrometastases were defined by the presence of isolated tumor cells or tumor cell clusters, detected either with HE staining and measuring less than $0.2 \mathrm{~mm}$ along the largest diameter, or using IHC. According to current recommendations, these occult LN metastases were classified as pNO.

Data analysis. Detection rates of SLNs with blue dye and ICG-FI techniques were calculated and compared. The sensitivity, specificity, and accuracy indexes for nodal staging were calculated for both techniques. The sensitivity, specificity, and accuracy were calculated using the standard definitions. Statistical significance was obtained with a Chi-square test and a $p$-value of less than 0.05 was considered significant. Agreement between the two techniques was evaluated by computing the kappa coefficient (к) with its $95 \%$ confidence interval (CI).

\section{Results}

Patients. From September 2013 to November 2015, 20 patients were included in the study. Clinical and pathological characteristics of the 20 patients are detailed in Table I. There were 11 females and nine males, with a median age of 70.5 years (range $=43-87$ years). The median body mass index (BMI) was $26.3 \mathrm{~kg} / \mathrm{m}^{2}$ (range $=18.8-36.3 \mathrm{~kg} / \mathrm{m}^{2}$ ). Fourteen patients presented with pT3-4 tumors and six with pTis-1-2 tumors.

Patients' nodal status, $L N$ and SLN characteristics, blue dye and fluorescent SLN detection rates. Regional LN metastases were identified in seven patients (35\%) by standard HE histopathological examination $(\mathrm{pN}+)$. The median number of 


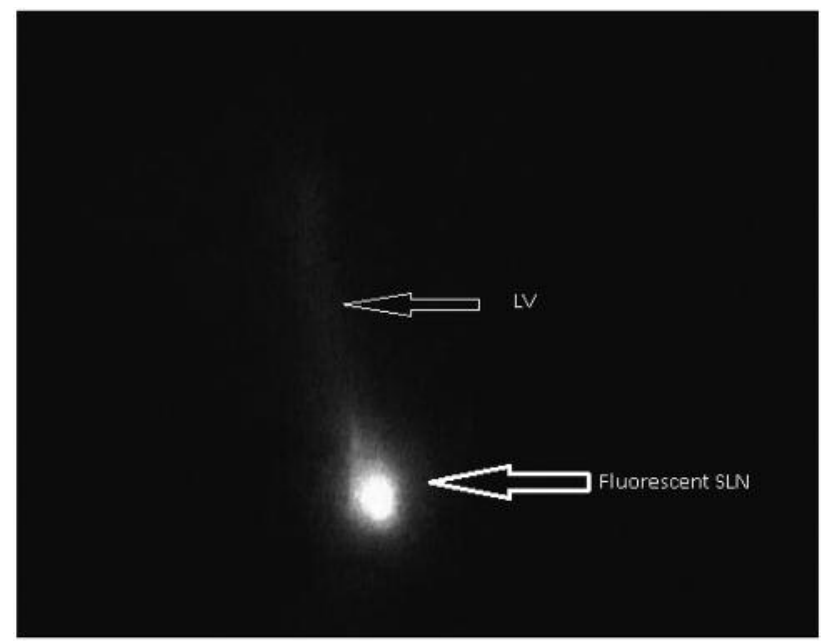

Figure 1. Fluorescent lymphatic vessel (LV) and SLN.

LNs retrieved per patient was 25 (range=5-41). The mean number of SLNs identified ex vivo by the two techniques was similar $(p=0.78)$. At pathology, the mean number of LNs stained with blue dye was 8.8 as compared to 6.5 for ICGFI (Table II). The SLN detection rate was 95\% (19/20 patients) with both blue dye and ICG-FI techniques. In one patient, no SLN was detected with either of the techniques. Of note, no technical problem was encountered at the time of dye injection in this case.

Sensitivity, specificity and accuracy of SLN detection. The sensitivity, specificity, and accuracy were $43 \%$ (3/7), 100\% (9/9), and $79 \%(15 / 19)$, respectively, for the blue dye technique, and $57 \%(4 / 7), 100 \%$ (11/11), and $84 \%$ (16/19), respectively, for the fluorescent technique (Table III). Two patients had fluorescentpositive SLNs that were also detected by blue dye afterwards at pathology. Among the four blue dye technique false-negative SLNs and the three FI false-negative SLNs (Table IV), two $(50 \%)$ and three $(75 \%)$, respectively, were found to be blue and fluorescent at pathology. Notably, three false-negative results occurred in the same patients with both techniques. All tumors were locally advanced (pT3 or pT4) tumors. Furthermore concerning false-negative results, two patients had only one positive $\mathrm{LN}$ at pathology and both had blue dye $(0 / 2)$ and fluorescent (0/1) SLN negativity intraoperatively. However, in one patient, the involved LN was fluorescent at pathology. The correlation between the two techniques (K test) was $83 \%$ (95\% $\mathrm{CI}=50.0-100 \%$ ). The sensitivity of the fluorescent technique was superior to that of the blue dye technique in patients with BMI $>25 \mathrm{~kg} / \mathrm{m}^{2}$, at $100 \%$ and $50 \%$, respectively.

SLN serial sectioning (authentic micrometastases). Among the 13 patients classified as node-negative at pathology
Table I. Patient and tumor characteristics.

\begin{tabular}{llc}
\hline Characteristic & & \\
\hline Patient age (years) & Median & 70.5 \\
Gender (n) & Range & $43-87$ \\
& Male & 9 \\
BMI $\left(\mathrm{kg} / \mathrm{m}^{2}\right)$ & Female & 11 \\
& Median & 26.3 \\
Location of primary tumor (n) & Range & $18.8-36.3$ \\
& Right colon & 9 \\
& Transverse colon & 1 \\
& Left colon & 4 \\
AJCC pTN (n) & Sigmoid colon & 6 \\
& TisN0 & 2 \\
& T1N0 & 2 \\
& T2N0 & 1 \\
& T3N0 & 6 \\
& T4aN0 & 2 \\
& T1N1 & 1 \\
Tumor size (cm) & T3N1 & 4 \\
& T4aN1 & 2 \\
& Median & 3.9 \\
& Range & $0-10$ \\
\hline
\end{tabular}

BMI: Body mass index; AJCC: American Joint Committee on Cancer.

Table II. Lymph node (LN) and sentinel lymph node (SLN) characteristics.

\begin{tabular}{lc}
\hline Characteristic & Mean (median; range) \\
\hline Number of LN resected & $22.4(25 ; 5-41)$ \\
Number of SLN detected in the OR & $1.4(1 ; 0-4)$ \\
BD technique & $1.5(1 ; 0-4)$ \\
FI technique & \\
Number of marked LN at pathology & $8.8(7 ; 1-17)$ \\
Blue & $6.5(7 ; 1-17)$ \\
Fluorescent &
\end{tabular}

OR: Operating room; BD: blue dye; FI: fluorescence imaging.

(pN0), further serial sectioning with HE staining of blue and fluorescent SLN did not up-stage these patients to authentic micrometastases. Serial sectioning with HE analysis of falsenegative SLNs by blue dye $(n=3)$ and fluorescence $(n=1)$ in positive-node staged $(\mathrm{pN}+)$ patients did not allow for correction of nodal staging to positive.

IHC staining of serially sectioned SLN (occult micrometastases). Among the 13 patients classified as nodenegative at pathology (pN0) by classical analyses (bivalve, $\mathrm{HE}$ ) and serial sectioning with $\mathrm{HE}$ staining, further pathological analysis by IHC on fluorescent and blue dye SLN allowed up-staging of three $(23 \%)$ and no patient $(0 \%)$, respectively. As these tumoral deposits were not detected by 
Table III. Crosstable for sensitivity, specificity, and accuracy calculation.

\begin{tabular}{|c|c|c|c|c|c|c|c|c|c|}
\hline \multirow[t]{2}{*}{ Blue SLN } & \multicolumn{3}{|c|}{$\mathrm{pN}(\mathrm{H} \& \mathrm{E}), \mathrm{n}$} & \multirow[t]{2}{*}{ Total, $\mathrm{n}$} & \multirow[t]{2}{*}{ Fluorescent SLN, n } & \multicolumn{3}{|c|}{$\mathrm{pN}(\mathrm{H} \& \mathrm{E}), \mathrm{n}$} & \multirow[t]{2}{*}{ Total, $\mathrm{n}$} \\
\hline & & 0 & 1 & & & & 0 & 1 & \\
\hline & 0 & 13 & 4 & 17 & & 0 & 13 & 3 & 16 \\
\hline \multirow[t]{3}{*}{$\mathrm{pN}(\mathrm{H} \& \mathrm{E})$} & 1 & 0 & 3 & 3 & $\mathrm{pN}(\mathrm{H} \& \mathrm{E})$ & 1 & 0 & 4 & 4 \\
\hline & & 13 & 7 & $20^{\mathrm{a}}$ & & & 13 & 7 & $20^{\mathrm{a}}$ \\
\hline & 0 & 13 & 4 & 17 & & 0 & 13 & 3 & 16 \\
\hline \multirow[t]{3}{*}{ pN (serial section) } & 1 & 0 & 3 & 3 & $\mathrm{pN}$ (serial section) & 1 & 0 & 4 & 4 \\
\hline & & 13 & 7 & $20^{\mathrm{a}}$ & & & 13 & 7 & $20^{\mathrm{a}}$ \\
\hline & 0 & 13 & 4 & 17 & & 0 & 10 & - & - \\
\hline \multirow[t]{2}{*}{ pN (IHC) } & 1 & 0 & 3 & 3 & pN (IHC) & 1 & 3 & - & - \\
\hline & & 13 & 7 & $20^{\mathrm{a}}$ & & & 13 & 7 & $20^{\mathrm{a}}$ \\
\hline
\end{tabular}

SLN: Sentinel lymph node; H\&E: hematoxilin and eosin; IHC: immunohistochemistry. aIncluding the patient with unsuccessful SLN detection by both techniques.

Table IV. Sentinel lymph node (SLN) staging results according to tumor stage ( $p T$ ).

\begin{tabular}{|c|c|c|c|c|c|c|c|c|c|c|c|}
\hline \multirow[b]{3}{*}{ Tumoral (pT) staging } & & & & \multicolumn{8}{|c|}{ SLN technique } \\
\hline & \multicolumn{3}{|c|}{ Patient nodal staging, $\mathrm{n}$} & \multicolumn{4}{|c|}{ Blue SLN, n } & \multicolumn{4}{|c|}{ Fluorescent SLN, n } \\
\hline & & pN0 & $\mathrm{pN} 1$ & $\mathrm{TN}$ & $\mathrm{FN}$ & $\mathrm{TP}$ & $\mathrm{FP}$ & $\mathrm{TN}$ & $\mathrm{FN}$ & $\mathrm{TP}$ & FP \\
\hline & pTis & 2 & 0 & 2 & 0 & - & - & 2 & 0 & - & - \\
\hline & pT1 & 2 & 1 & 2 & 0 & 1 & 0 & 2 & 0 & 1 & 0 \\
\hline & pT2 & 1 & 0 & 1 & 0 & - & - & 1 & 0 & - & - \\
\hline & pT3 & 6 & 4 & $6\left(8^{a}\right)$ & $3\left(1^{\mathrm{a}}\right)$ & $0\left(2^{\mathrm{a}}\right)$ & 0 & $6^{\mathrm{b}}$ & $2\left(0^{\mathrm{a}}\right)$ & $2\left(4^{a}\right)$ & 0 \\
\hline & pT4a & 2 & 2 & 2 & $1\left(1^{a}\right)$ & $1\left(1^{a}\right)$ & 0 & 2 & 1 & 1 & 0 \\
\hline
\end{tabular}

pN0: Negative nodal staging at pathology; pN1: positive nodal staging at pathology; TN: true-negative; FN: false-negative; TP: true-positive; FP: false-positive. ancluding blue LN and fluorescent LN found afterwards at pathology; bincluding three patients with occult micrometastases.

the initial serial sectioning, they were classified as occult micrometastases.

\section{Discussion}

ICG-FI has emerged as a promising new imaging technique for in vivo detection of SLNs of colorectal origin $(13,14)$. FI may offer better tissue contrast, facilitating detection in deep anatomic regions such as the abdomen, and is easy and safe to perform $(13,14,17)$. Furthermore, ICG-FI is not influenced by the patient's BMI nor by lymphatic invasion, as has been shown for conventional methods, and has been shown to increase SLN detection in comparison with standard techniques $(16,18)$.

Although the ex vivo blue dye technique in SLN detection in CRC is well documented in the literature and has been demonstrated to be as effective and sensitive as the in vivo technique $(19,20)$, no study has yet evaluated the role of ICG-FI using the ex vivo technique for SLN detection.
Potential advantages of the ex vivo approach are its ease of use, the fact that it does not prolong the surgical procedure, and that it avoids the risk, although exceptional, of allergic reaction after intravenous injection.

Our study shows that the ICG-FI technique is feasible with an SLN detection rate of 95\%. However, the sensitivities reported here with the classical blue dye and with ICG-FI of $43 \%$ and $57 \%$, respectively, are low and not sufficiently predictive of nodal status. As a minimal dissection of the mesentery is often necessary to identify blue lymphatic channels and blue LNs (19), we hypothesize that some of the failures were related to our technique, which avoided any dissection of the mesentery during the ex vivo procedure. This is consistent with the finding that in two patients with fluorescent SLN and no blue SLN found on $e x$ vivo exploration in the operating room, we were able to eventually demonstrate blue dye staining at examination in the Pathology Department. This is a critical point of our study. However, we observed that using ICG-FI for SLN 
detection after the use of blue dye did not seem to alter the detection of fluorescent SLN. Another potential explanation for the high false-negative rates in our study is the inclusion of patients with locally advanced tumors. Seventy percent of included patients had pT3-4 tumors. This could cause a higher risk of missed metastases due to lymphatic channel blockages (20). This is problematic as these patients are probably the most at-risk for developing further metastatic disease. Despite these disappointing results, we found that ICG-FI was more sensitive than the classical blue dye technique in patients with $\mathrm{BMI}>25 \mathrm{~kg} / \mathrm{m}^{2}$, consistent with a previous report (16). Additionally, we limited the identification of SLNs to those appearing fluorescent after ex vivo ICG injection in the operating room. This is a shorter period than was used in a previous study which defined SLNs as all fluorescent LNs identified ex vivo after in vivo injection of ICG (13). If we analyze our results in the same way (fluorescent LNs detected in Pathology Department), in fact, we greatly increase the sensitivity rate to $90 \%$.

Such differences in the methodologies used and in their interpretation underline the need for a better definition of the concept of SLN in CRC. In contrast with melanoma and breast cancer, where the SLN is defined as the first anatomical relay for metastasis through lymphatic dissemination, SLNs detected by FI in CRC could be defined as a larger concept that includes lymphatic channels draining to a 'hyperactive' LN network that is suspected to be more likely to harbor LN metastases. This could be less effective than the current SLN concept used in breast cancer and melanoma but still allow for the analysis of a limited number of LNs with advanced pathological techniques (serial section, IHC, and RT-PCR) in colorectal cancer.

None of the patients with negative nodal status by standard analyses were up-graded by the analysis of blue dye and fluorescent SLN by serial sectioning. These results are inferior to those reported in the literature, where approximately $7-15 \%$ of patients were up-graded after HE staining of serial sections of SLNs (19). Conversely, three patients $(27 \%)$ and no patient were upgraded by IHC of SLNs detected by the ICG-FI and by the blue dye technique, respectively. These results are consistent with data in the literature reporting a proportion of up-graded patients of from $7 \%$ to $31 \%(6,21)$. The prognostic value of occult metastases detected by IHC or RT-PCR in colorectal cancer remains a matter of debate (22-24). The results of an ongoing study that will address this issue are expected (25). As recommended in the AJCC classification, the tumor stage of these patients was not modified and they did not receive adjuvant chemotherapy.

In this preliminary study, the ex vivo injection of ICG to identify SLNs in patients with CRC appears to be a valuable approach, superior to the classical blue dye technique in patients with high BMI. Moreover, focused pathological examination of these SLNs using IHC allowed identification of occult micrometastases in a quarter of the patients examined. However, in our experience, the sensitivity of ICG-FI in SLN detection remains low and is associated with a high rate of false-negatives, highlighting the need for further technical refinements to precisely define the methods for injection and the kinetics for examination.

\section{Acknowledgements}

This study was supported, in part, by a grant from Les Amis de Bordet who support research in oncology, and by the R\&D Clinical Applications of Fluorescence Imaging Group (Coordinator: Professor Pierre Bourgeois). The funding sources did not have any role in study design, data collection, interpretation of data, or the decision to submit the manuscript for publication. The Authors acknowledge the contribution of a medical writer, Sandy Field, Ph.D., to this article.

\section{References}

1 André T, de Gramont A, Vernerey D, Chibaudel B, Bonnetain F, Tijeras-Raballand A, Scriva A, Hickish T, Tabernero J, Van Laethem JL, Banzi M, Maartense E, Shmueli E, Carlsson GU, Scheithauer W, Papamichael D, Möehler M, Landolfi S, Demetter P, Colote S, Tournigand C, Louvet C, Duval A, Fléjou $\mathrm{JF}$, and de Gramont A: Adjuvant fluorouracil, leucovorin, and oxaliplatin in stage II to III colon cancer: updated 10-year survival and outcomes according to BRAF mutation and mismatch repair status of the MOSAIC study. J Clin Oncol 33: 4176-4187, 2015.

2 Edge SB, Byrd DR and Compton CC: AJCC Cancer Staging Manual, Seventh Edition. Springer, New York, 2010.

3 Figueredo A, Coombes ME and Mukherjee S: Adjuvant therapy for completely resected stage II colon cancer. Cochrane Database Syst Rev 3: CD005390, 2008.

4 Dummer R, Hauschild A, Guggenheim M, Jost L, Pentheroudakis $\mathrm{G}$ and ESMO Guidelines Working Group: Melanoma: ESMO Clinical Practice Guidelines for diagnosis, treatment and follow-up. Ann Oncol 21: v194-v197, 2010.

5 Lyman GH, Temin S, Edge SB, Newman LA, Turner RR, Weaver DL, Benson AB 3rd, Bosserman LD, Burstein HJ, Cody H 3rd, Hayman J, Perkins CL, Podoloff DA and Giuliano AE: American Society of Clinical Oncology Clinical Practice. Sentinel lymph node biopsy for patients with early-stage breast cancer: American Society of Clinical Oncology clinical practice guideline update. J Clin Oncol 32: 1365-1383, 2014.

6 Bilchik A, Saha S, Wiese D, Stonecypher JA, Wood TF, Sostrin S, Turner RR, Wang HJ, Morton DL and Hoon DS: Molecular staging of early colon cancer on the basis of sentinel node analysis: a multicenter phase II trial. J Clin Oncol 19: 3238-3244, 2001.

7 van der Pas MHGM, Meijer S, Hoekstra OS, Riphagen II, de Vet HC, Knol DL, van Grieken NC and Meijerink WJ: Sentinellymph-node procedure in colon and rectal cancer: a systematic review and meta-analysis. Lancet Oncol 12: 540-550, 2011.

8 Nicastri DG, Doucette JT, Godfrey TE and Hugues SJ: Is occult lymph node disease in colorectal cancer patients clinically significant? A review of the relevant literature. J Mol Diagn 9: 563-571, 2007. 
9 Sloothaak DAM, Sahami S, van der Zaag-Loonen HJ van der Zaag ES, Tanis PJ, Bemelman WA and Buskens CJ: The prognostic value of micrometastases and isolated tumours cells in histologically negative lymph nodes of patients with colorectal cancer: a systematic review and meta-analysis. Eur J Surg Oncol 40: 263-269, 2014.

10 Hirche C, Murawa D, Mohr Z, Kneif S and Hünerbein M: ICG fluorescence-guided sentinel node biopsy for axillary nodal staging in breast cancer. Breast Cancer Res Treat 121: 373-378, 2010 .

11 Gilmore DM, Khullar OV, Gioux S, Stockdale A, Frangioni JV, Colson YL and Russell SE: Effective low-dose escalation indocyanine green for near-infrared fluorescent sentinel lymph node mapping in melanoma. Ann Surg Oncol 20: 2357-2363, 2013.

12 Rossi EC, Ivanova A and Boggess JF: Robotically assisted fluorescence-guided lymph node mapping with ICG for gynecologic malignancies: a feasibility study. Gynecol Oncol 124: 78-82, 2012.

13 Kusano M, Tajima Y, Yamazaki K, Kato M, Watanabe M and Miwa M: Sentinel Node Mapping Guided by Indocyanine Green Fluorescence Imaging: A New Method for Sentinel Node Navigation Surgery in Gastrointestinal Cancer. Dig Surg 25: 103-108, 2008.

14 Hirche C, Mohr Z, Kneif S, Doniga S, Murawa D, Strik M and Hünerbein M: Ultrastaging of colon cancer by sentinel node biopsy using fluorescence navigation with indocyanine green. Int J Colorectal Dis 7: 319-324, 2012.

15 Noura S, Ohue M, Seki Y, Tanaka K, Motoori M, Kishi K, Miyashiro I, Ohigashi H, Yano M, Ishikawa O and Miyamoto Y: Feasibility of a lateral region sentinel node biopsy of lower rectal cancer guided by indocyanine green using a near-infrared camera system. Ann Surg Oncol 17: 144-151, 2010.

16 Nagata K, Endo S, Hidaka E, Tanaka J, Kudo SE and Shiokawa A: Laparoscopic sentinel node mapping for colorectal cancer using infrared ray laparoscopy. Anticancer Res 26: 2307-2312, 2006.

17 Kitagawa Y, Fujii H, Kumai K, Kubota T, Otani Y, Saikawa Y, Yoshida M, Kubo A and Kitajima M: Recent advances in sentinel node navigation for gastric cancer: a paradigm shift of surgical management. J Surg Oncol 90: 147-151, 2005.
18 Bembenek AE, Rosenberg R, Wagler E, Gretschel S, Sendler A, Siewert JR, Nährig J, Witzigmann H, Hauss J, Knorr C, Dimmler A, Gröne J, Buhr HJ, Haier J, Herbst H, Tepel J, Siphos B, Kleespies A, Koenigsrainer A, Stoecklein NH, Horstmann O, Grützmann R, Imdahl A, Svoboda D, Wittekind C, Schneider W, Wernecke KD and Schlag PM: Sentinel lymph node biopsy in colon cancer: a prospective multicenter trial. Ann Surg 245: 858-863, 2007.

19 Liberale G, Lasser P, Sabourin JC, Malka D, Duvillard P, Elias D, Boige V, Goéré D, Ducreux M and Pocard M: Sentinel lymph nodes of colorectal carcinoma: reappraisal of 123 cases. Gastroenterol Clin Biol 31: 281-285, 2007.

20 Oh SY, Kim DY, Kim YB and Suh KW. Clinical application of sentinel lymph node mapping in colon cancer: in vivo vs. ex vivo techniques. Ann Surg Treat Res 87: 118-122, 2014.

21 Wood TF, Saha S, Morton DL, Tsioulias GJ, Rangel D, Hutchinson W Jr, Foshag LJ and Bilchik AJ: Validation of lymphatic mapping in colorectal cancer: in vivo, ex vivo, and laparoscopic techniques. Ann Surg Oncol 8: 150-157, 2001.

22 Greenson JK, Isenhart CE, Rice R, Mojzisik C, Houchens D and Martin EW Jr: Identification of occult micrometastases in pericolic lymph nodes of Duke's B colorectal cancer patients using monoclonal antibodies against cytokeratin and CC49. Correlation with long term survival. Cancer 73: 563-569, 1994.

23 Bukholm IR, Bondi J, Wiik P, Nesland JM, Andersen SN, Bakka $A$ and Bukholm G: Presence of isolated tumor cells in mesenteric lymph nodes predicts poor prognosis in patients with stage II colon cancer. Eur J Surg Oncol 29: 862-866, 2003.

24 Liefers GJ, Cleton-Jansen AM, van de Velde CJ, Hermans J, van Krieken JH, Cornelisse CJ and Tollenaar RA: Micrometastases and survival in stage II colorectal cancer. N Engl J Med 339: 223-228, 1998.

25 Lips DJ, Koebrugge B, Liefers GJ, van de Linden JC, Smit VT, Pruijt HF, Putter H, van de Velde CJ and Bosscha K: The influence of micrometastases on prognosis and survival in stage I-II colon cancer patients: the Enroute $\oplus$ Study. BMC Surgery 11: 11, 2011.

Received July 17, 2016

Revised August 1, 2016

Accepted August 4, 2016 